\title{
Effectiveness of Acceptance and Commitment Therapy and Logotherapy on Marital Satisfaction of Veterans' Spouses
}

\section{ART ICLE INF O}

\section{Article Type}

Original Research

\section{Authors}

Falahati M. ${ }^{1} M A$,

Shafiabady A.*2 $P h D$,

Jajarmi M. ${ }^{1} P h D$,

Mohamadipoor M. ${ }^{3} \mathrm{PhD}$

\section{A B S T R A C T}

Aims Marital satisfaction is one of the factors that can affect not only on the life quality of veterans but also on their families and society. The aim of this study was to investigate the effectiveness of acceptance and commitment therapy and logotherapy on marital satisfaction of veterans' spouses.

Materials \& Methods In this quasi-experimental research with pretest-posttest design, with two experimental groups and one control group, 60 people of veterans' spouses were studied in Tehran in 2017. They were selected by convenience sampling method and randomly assigned into acceptance and commitment therapy group, logotherapy group, and control group ( $\mathrm{n}=20$ in each group). Experimental groups were treated in 9 sessions- in 120 minutes, while the control group received no cure. The research tools were a demographic questionnaire and Enrich marital satisfaction questionnaire. Data were analyzed using SPSS 18 software, through one-way analysis of covariance and Tukey's post hoc test.

Findings By controlling the effects of pre-test scores, there was a significant difference between the mean scores of marital satisfaction of the groups in the posttest step $(F=6.041 ; p=0.004)$. The mean scores of marital satisfaction in the acceptance and commitment therapy group $(\mathrm{p}=0.023)$ and the logotherapy group $(\mathrm{p}=0.006)$ were significantly different in comparison with the control group. There was no significant difference between two experimental groups and both methods had the same effectiveness ( $\mathrm{p}=0.873$ ).

Conclusion Acceptance and commitment therapy and logotherapy are effective in increasing the marital satisfaction of veterans' spouses.

Keywords Acceptance and Commitment Therapy; Logotherapy; Marital; Satisfaction; Veterans; Spouses

\section{I T A T I O N L IN KS}

[1] An inquiry into the effect of teaching the skills ... [2] Investigating the influence of training ... [3] Effect of family problem-soving on ... [4] Intimacy, differentiation and personality ... [5] The relationship between marital ... [6] Research on the nature and determinants ... [7] Acceptance and commitment therapy ... [8] Randomized controlled trial of ... [9] The efficacy of acceptance and commitment ... [10] Psychotherapeutic interventions at the end ... [11] The meaning in life questionnaire: assessing ... [12] A review of acceptance and commitment ... [13] Using acceptance and commitment therapy to ... [14] Effectiveness of acceptance and commitment ... [15] Effectiveness of acceptanceand commitment ... [16] The role of exestential meaning as a buffer ... [17] LogotherapyEnhanced REBT: an integration ... [18] Disconnection, depression, and spirituality: A study of the role ... [19] The role of group counseling with logo-therapeutic approach ... [20] How are the experiences and needs of families ... [21] Evaluation of a psychoeducational group ... [22] Family psychoeducation and schizophrenia: a review ... [23] Effectiveness of acceptance and commitment ... [24] The importance of sample size and statistical ... [25] The Effect of educating about common changes in ... [26] Comparative study of the effect of group ... [27]. Predicting marital success with prepare ... [28] Effect of irrational thoughts (cognitive approach) ... [29] Effect of family therapy training on cognitive ... [30] Acceptance and commitment therapy, relational ... [31] Man's search for Meaning: An Introduction ... [32] . Effectiveness of group logotherapy ... [33] The doctor and the soul: from ... [34] Get out of your mind and into your ... [35] A practical guide to acceptance and ... [36] Climbing our hills: a beginning conversation on the comparison of acceptance and commitment therapy and traditional ... [37] Depression in youth: psychosocial ... [38] Marital cognitions and depression in the context of marital... [39] Theory and practice in group ...

\section{Article History \\ Received: January 2, 2019 \\ Accepted: May 15, 2019 \\ ePublished: July 21, 2019 \\ orrespondence t Highway, Tehran, Iran. Po \\ Fax: +98(21) 48393299 \\ ashafiabady@yahoo.com}

Education \& Psychology Faculty, Al- 


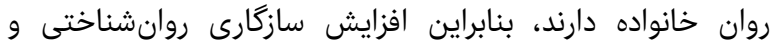

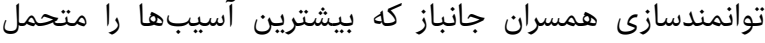

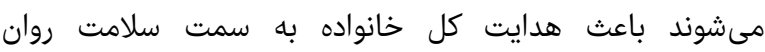

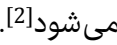

رضايت يك فرد از زندكى زناشويى، به منزله رضايت وى از خانوانوادواده

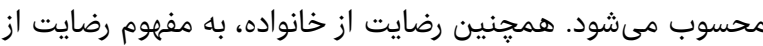

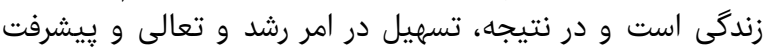

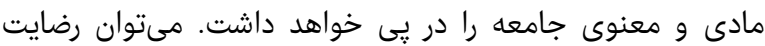

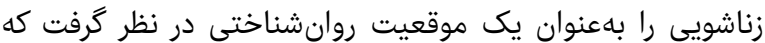

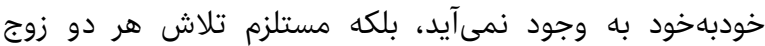

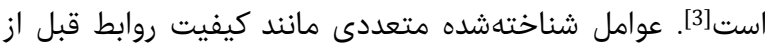

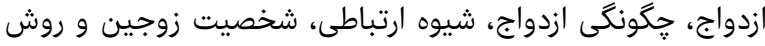

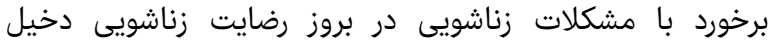

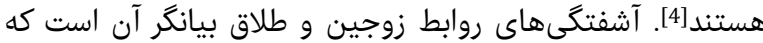

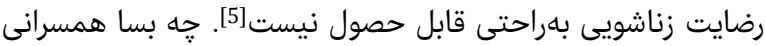

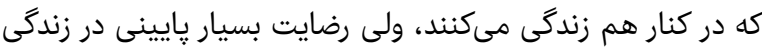

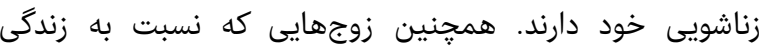

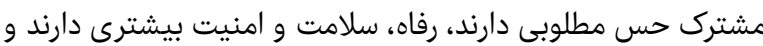

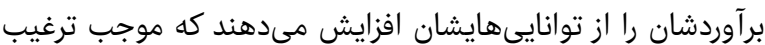

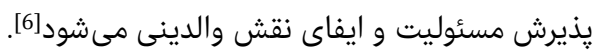

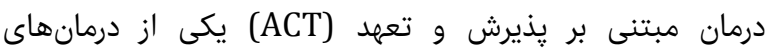

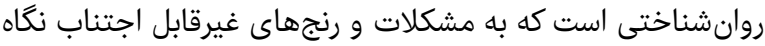

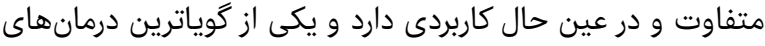

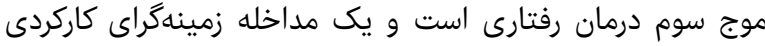

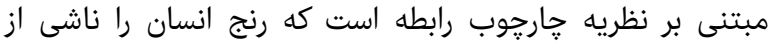

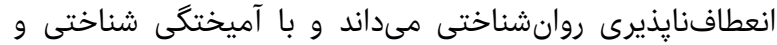

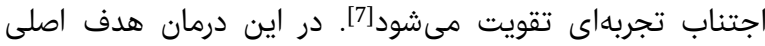

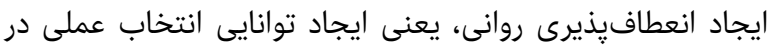

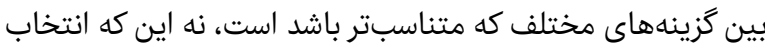

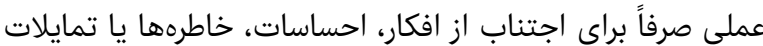

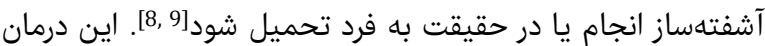

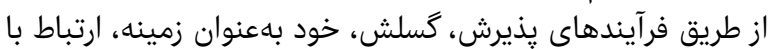

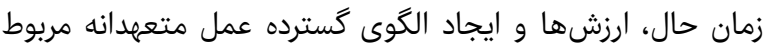

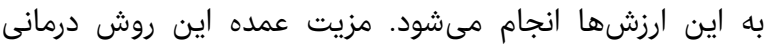

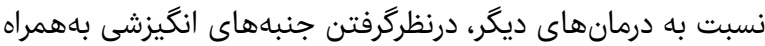

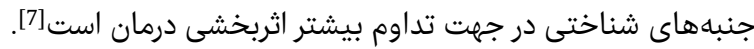

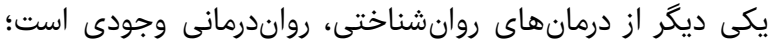

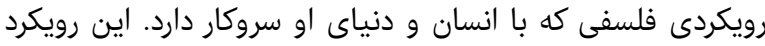

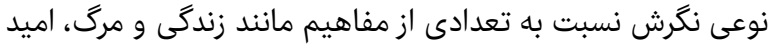

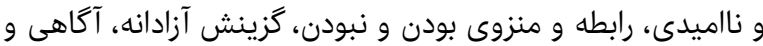

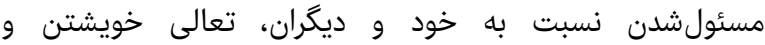

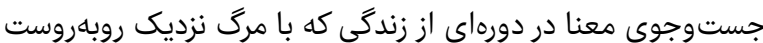

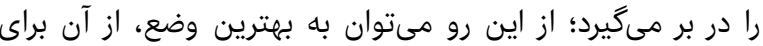

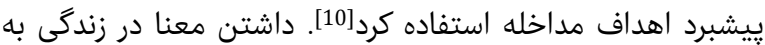

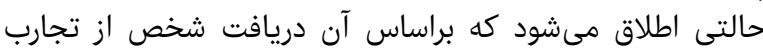

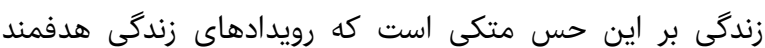

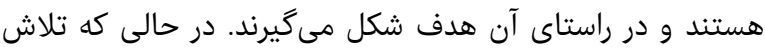

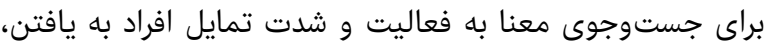

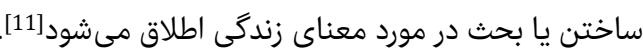

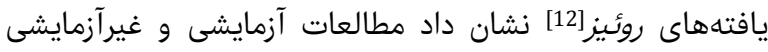

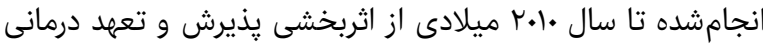

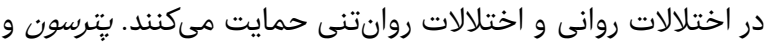

اثربخشى درمان مبتنى بر يذيرش و تعهد و معنادرمانى بر رضايت زناشويى همسران جانبازان

\author{
MA مريم فلاحتى \\ كروه روانشناسى، دانشكده علوم انسانى، واحد بجنورد، دانشگاه آزاد اسلامى،

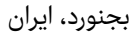

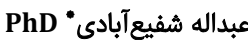

كروه مشاوره، دانشكده روانشهانهاسى و و علوم تربيتى، دانشكاه علامه طباطبايى،

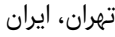

PhD محمود جاجرمى ايران كروه روانشناسى، دانشكده علوم انسانى، واحد بجنورد، دانشكاه آزاد اسلامى،

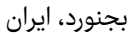

PhD محمد محمدى ائيورن كروه روانشناسى، دانشكده علوم انسانى، واحد قوحِان، دانشگاه آزاد اسلامى، قوخٍان، ايران

جكيده

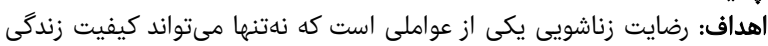

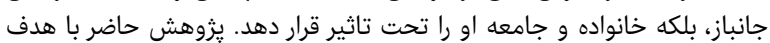

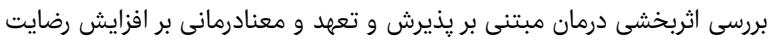

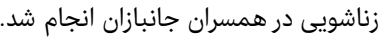

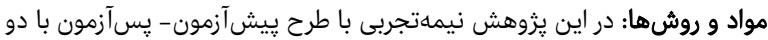

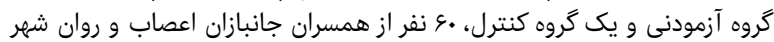

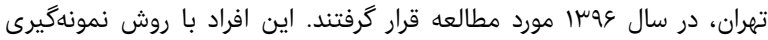

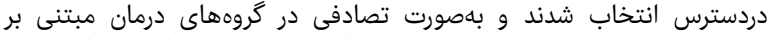

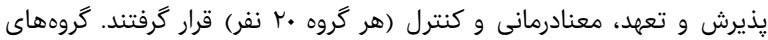

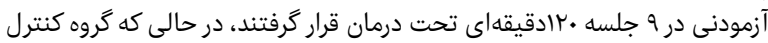

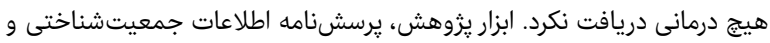

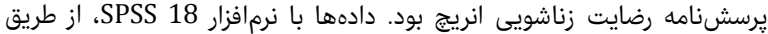

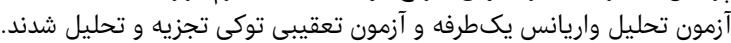

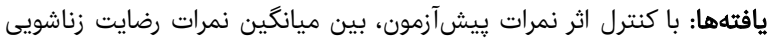

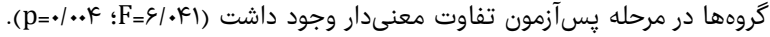

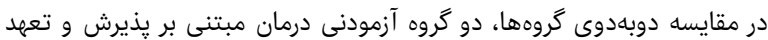

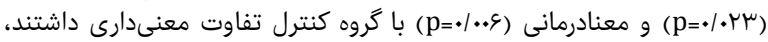

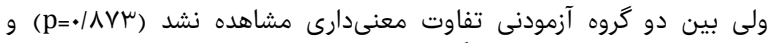

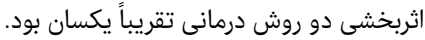

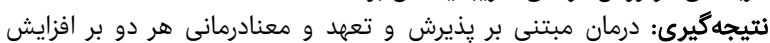

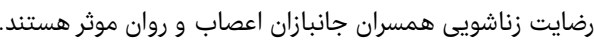

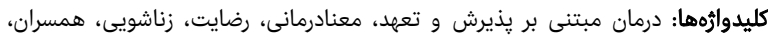
جانبازان

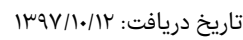

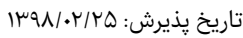

"نويسنده مسئول: تاريخ: ashafiabady@yahoo.com

\section{مقدمه - مقد}

با كذشت سالها از جنگ تحميلى، هنوز خانوادههاى جانبازان

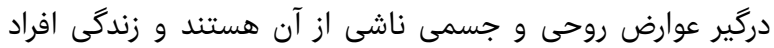

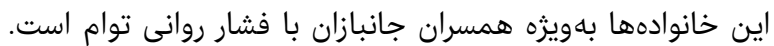

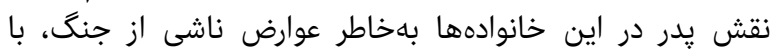

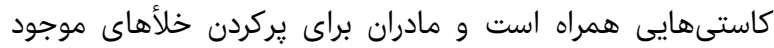

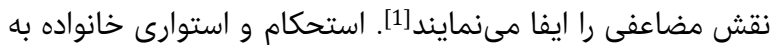

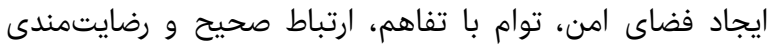

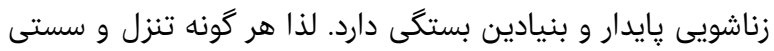

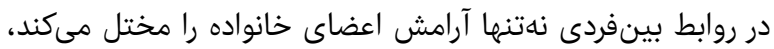

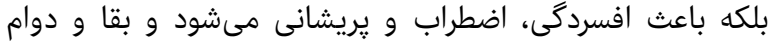

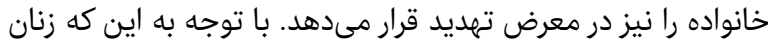

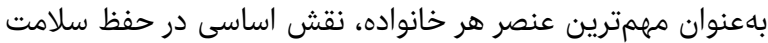




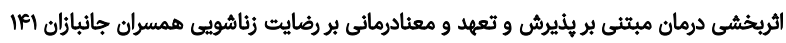

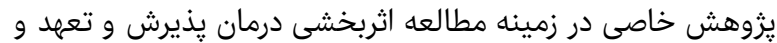

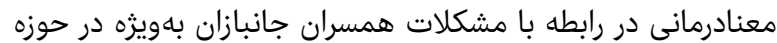

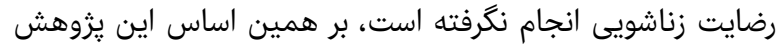

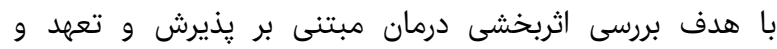

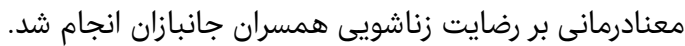

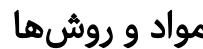

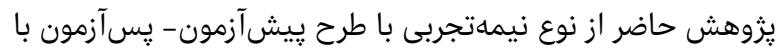

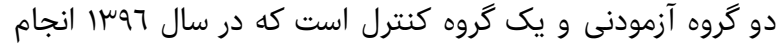

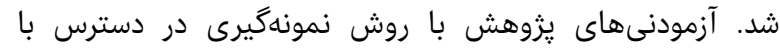

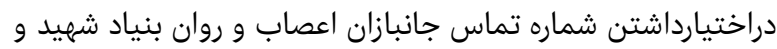

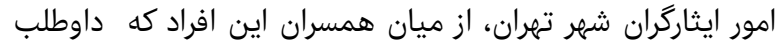

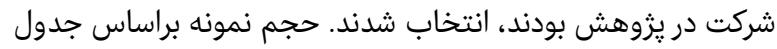

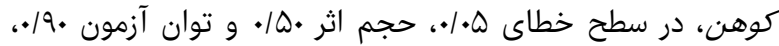

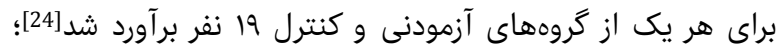

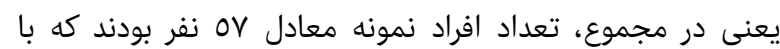

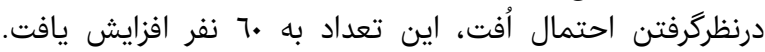

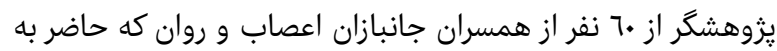

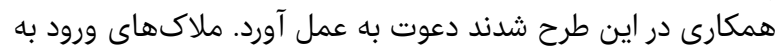

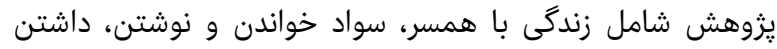

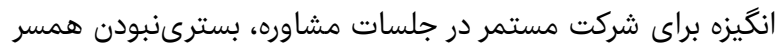

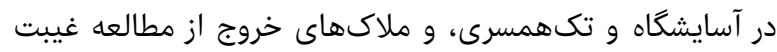

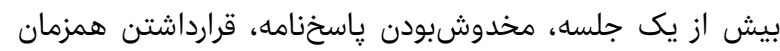

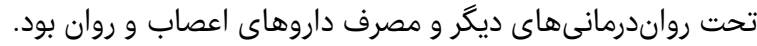

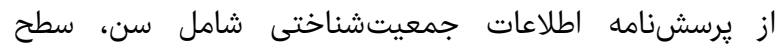

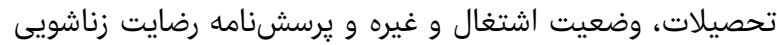

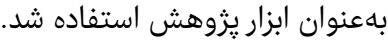

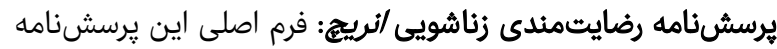

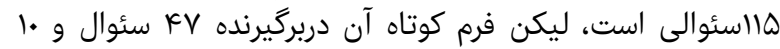

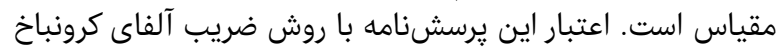

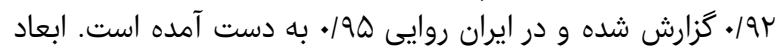

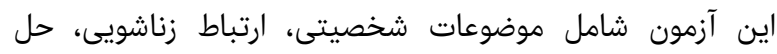

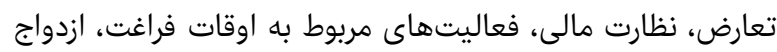

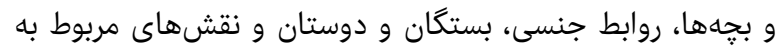

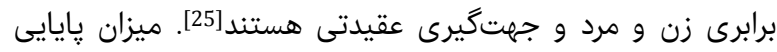

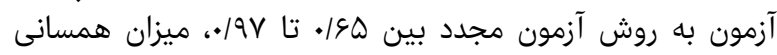

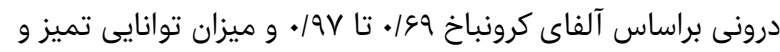

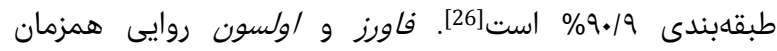

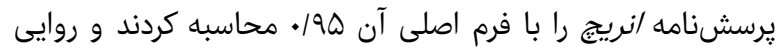

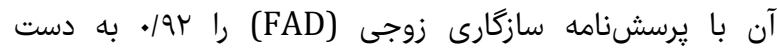

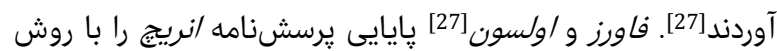

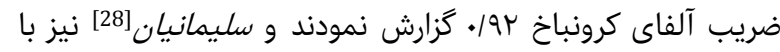

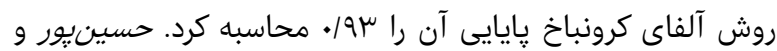

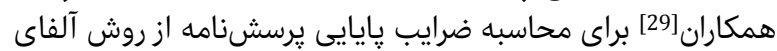

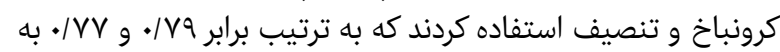
دست آمدون

بعد از هماهنگى با سازمان مربوطه و كسب مجوز و و جلب رضايت

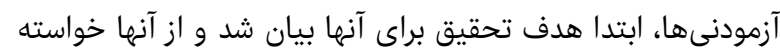

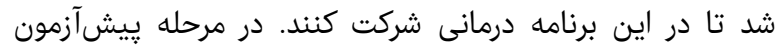

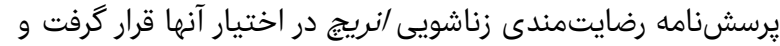

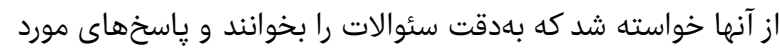

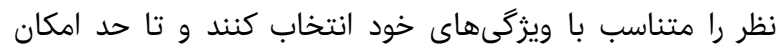

همكاران[13] نشان دادند كه درمان مبتنى بر يذيرش و تعهد در

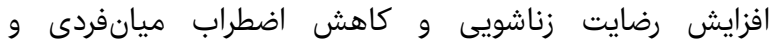

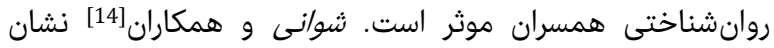

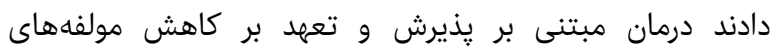

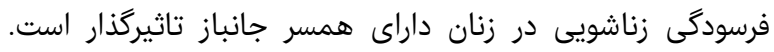

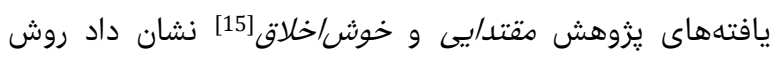

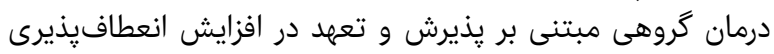

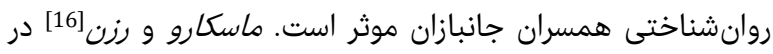

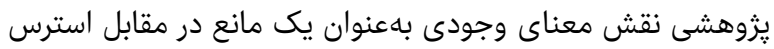

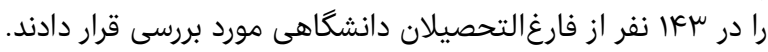

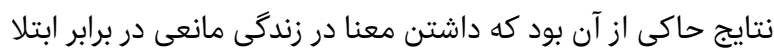

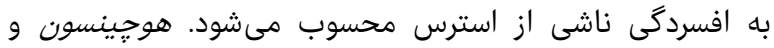

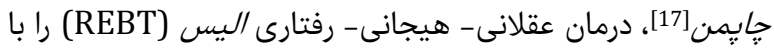

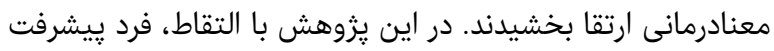

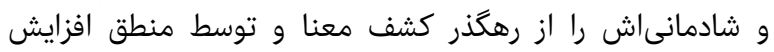

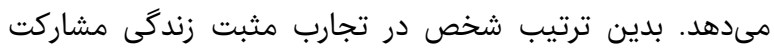

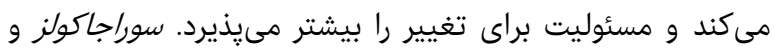

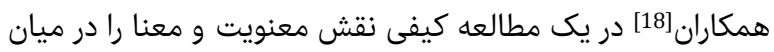

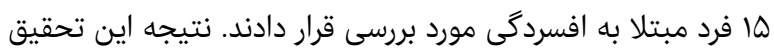

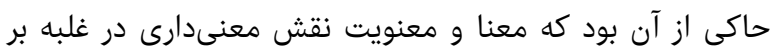

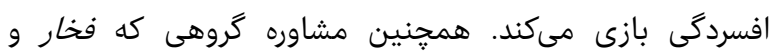

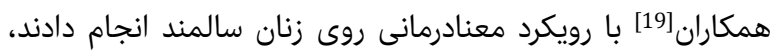

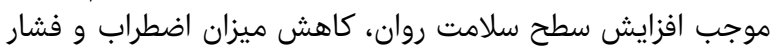

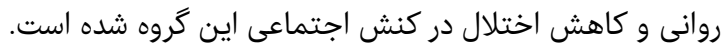

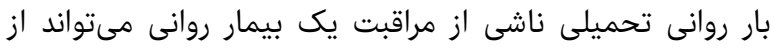

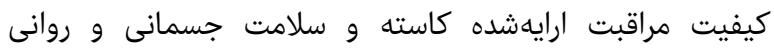

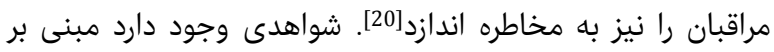

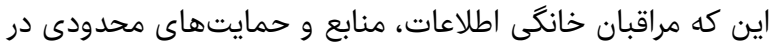

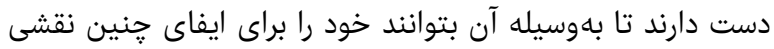

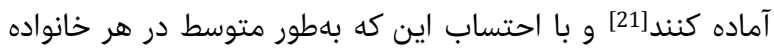

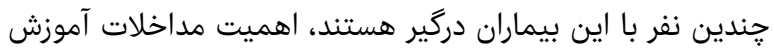

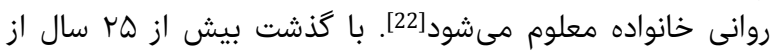

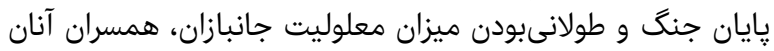

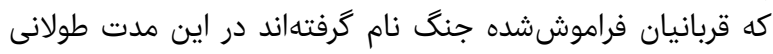

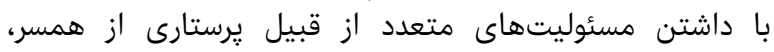

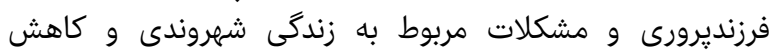

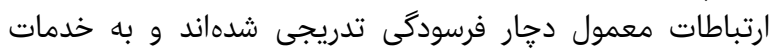

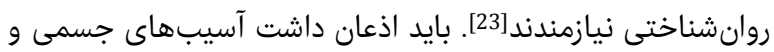

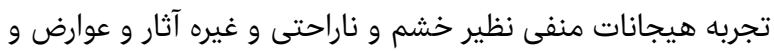

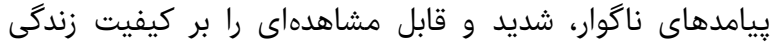

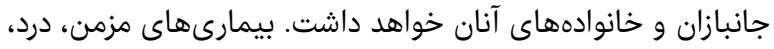

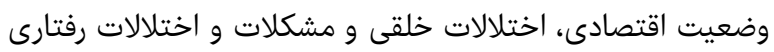

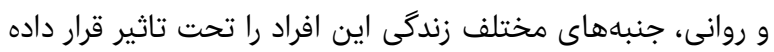

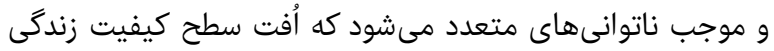

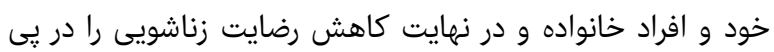

با توجه به اهميت مسايل و مشكلات جانبازان و همسران آنها،

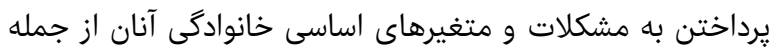

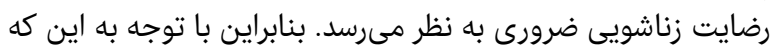

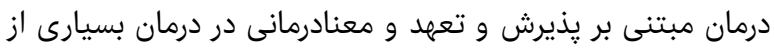

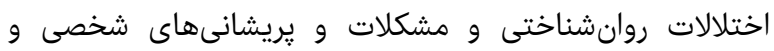

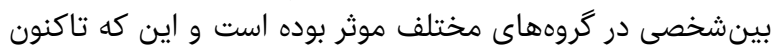


درمانى دريافت نكرد. يس از اتمام دوره آموزش براى گروههاى

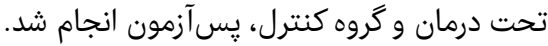

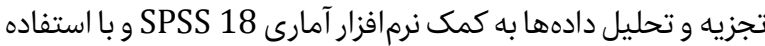

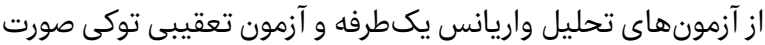

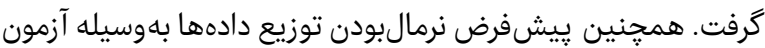
كولموكروف- اسميرنوف مورد بررسى و تاييد واقع شد. توند

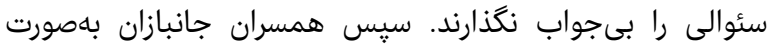

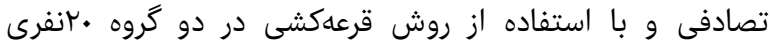

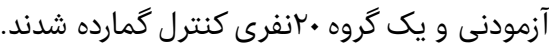

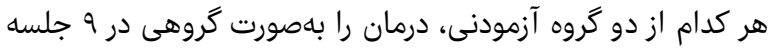

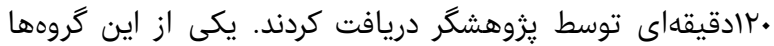

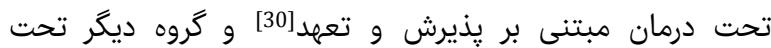

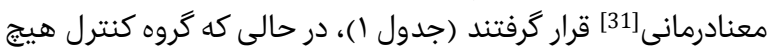

جدول () خلاصه محتواى جلسات درمانى اجراشده براى همسران جانبازان اعصاب و روان

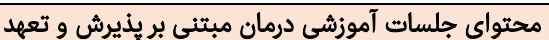

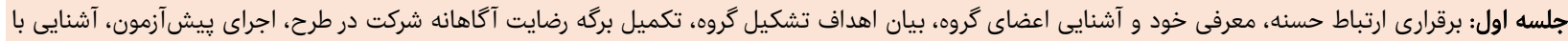

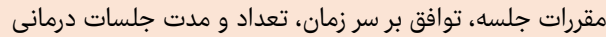

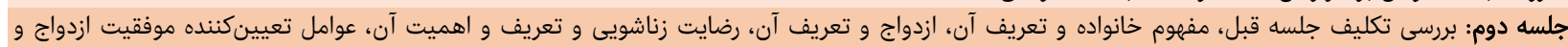

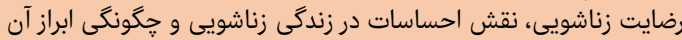

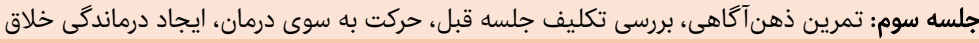

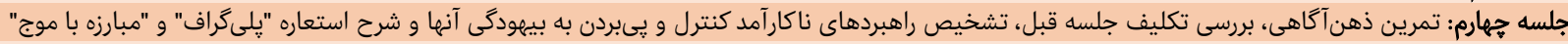

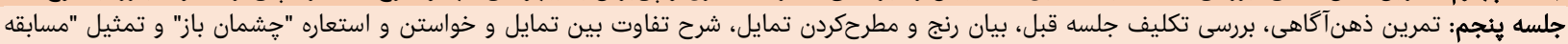

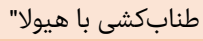
جلسه ششم: تمرين ذهن آكاهى، مطرحكردن هم آميزى شناختى (آميختگى با محتواى شناختى) و تغييردادن قواعد زبانى، تمرين "شير، شير، شير" و تمثيل "مسافران

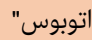

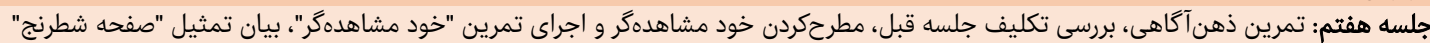

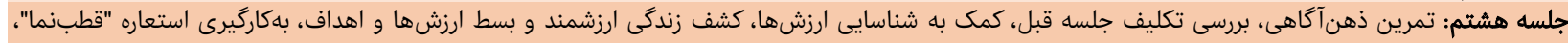

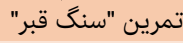

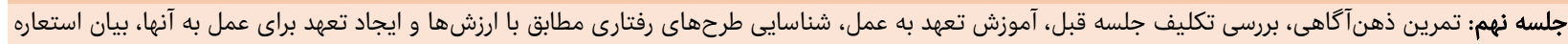

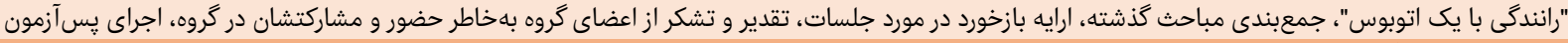

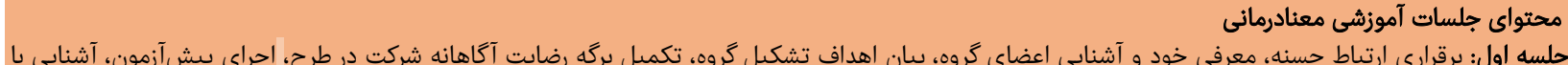

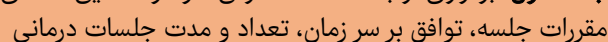

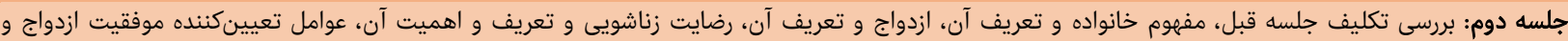

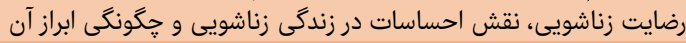

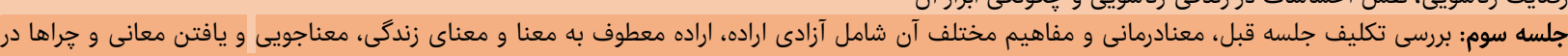

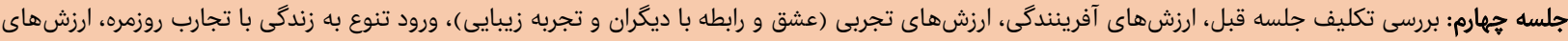

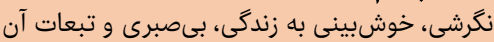

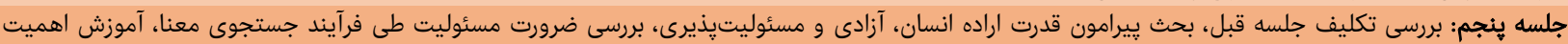

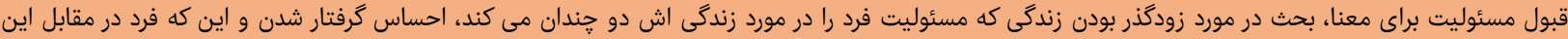

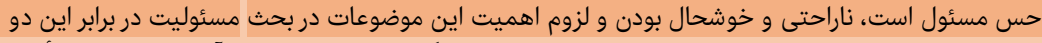

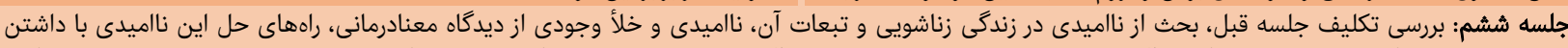

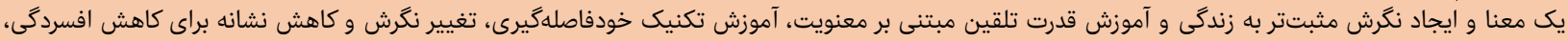

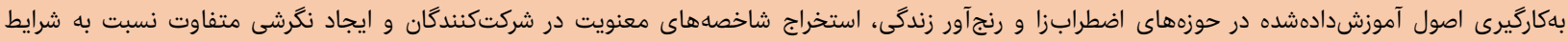

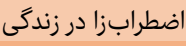

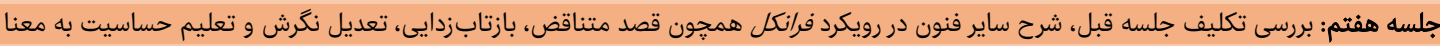

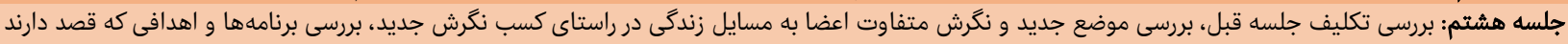

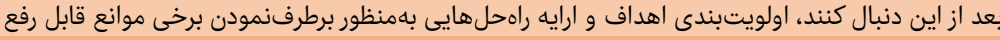

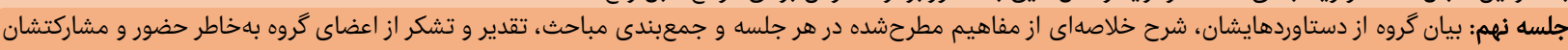

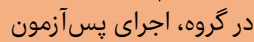

حيث وضعيت شغلى بيشتر افراد خانهدار بودند.

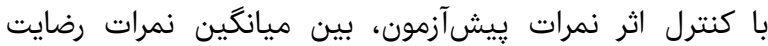

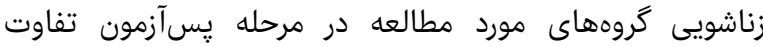

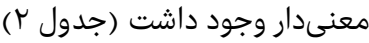

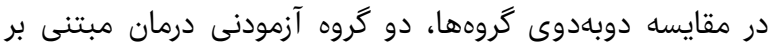

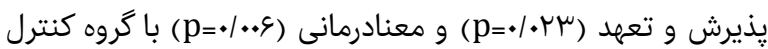

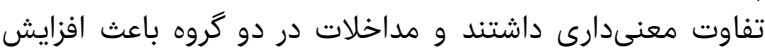

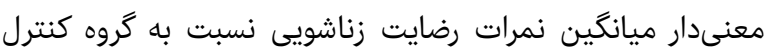

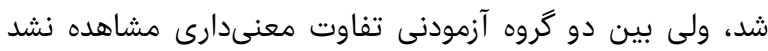

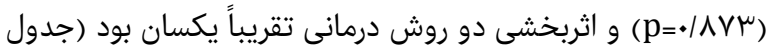

يافتهها

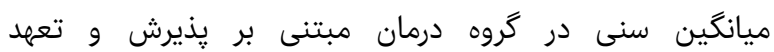
. گروه كنترل \$

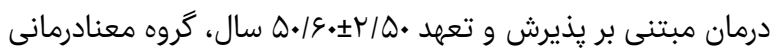
درم/

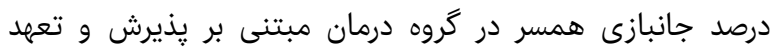
كنتر

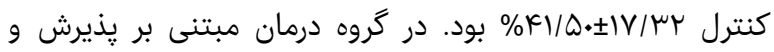

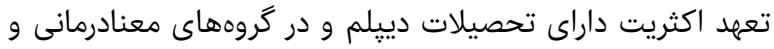

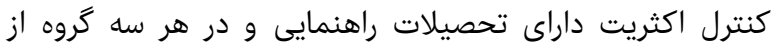




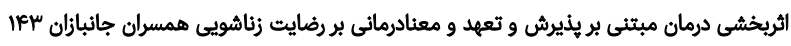

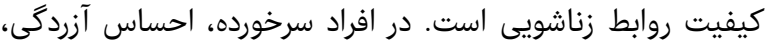

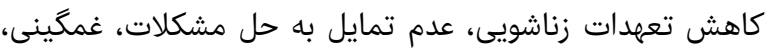

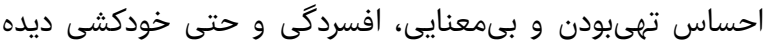
مى شىود [33]. نتايج يزوهش نشان دان داد كه دو مداخله مذكور بر افزايش رضايت

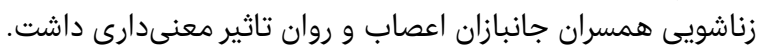

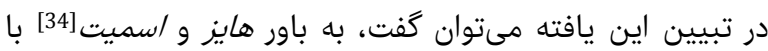

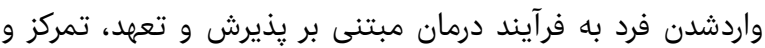

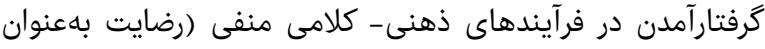

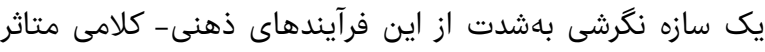

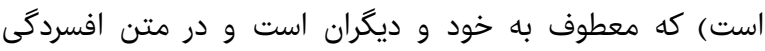

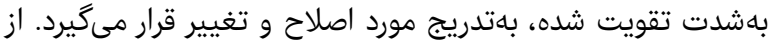

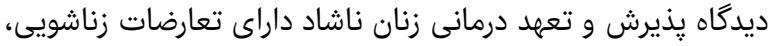

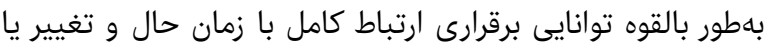

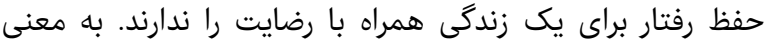

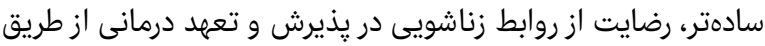

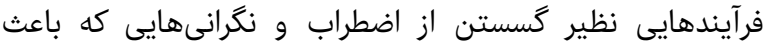

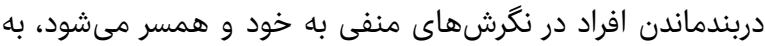

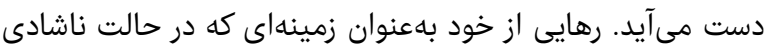

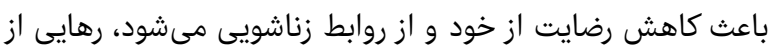

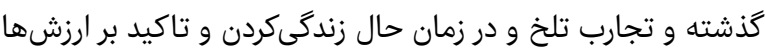

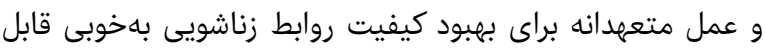

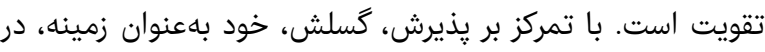

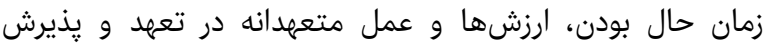

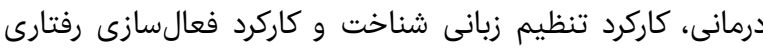

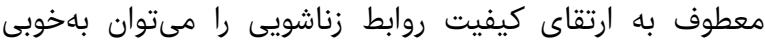

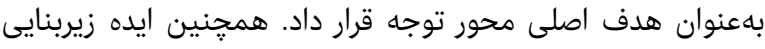

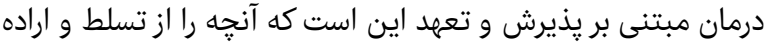

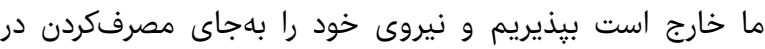

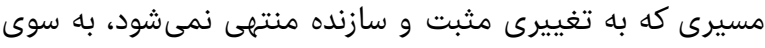

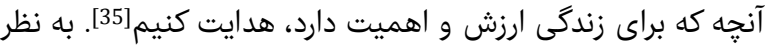

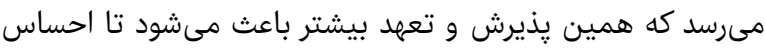

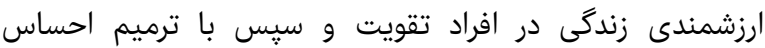

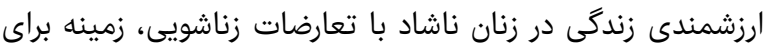

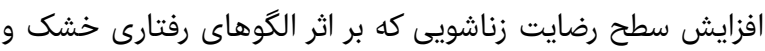

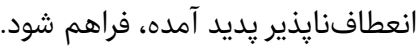

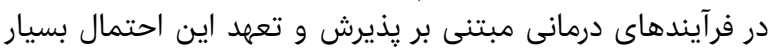

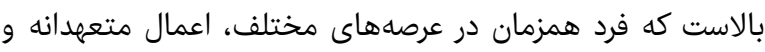

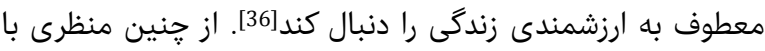

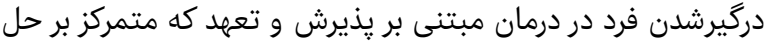

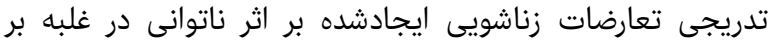

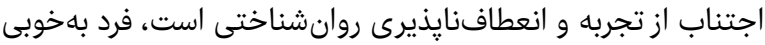

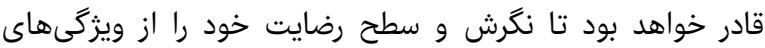

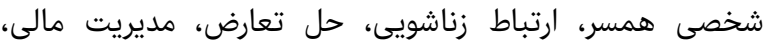

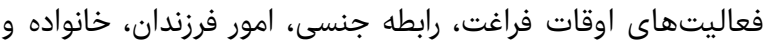

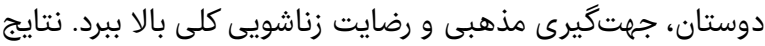

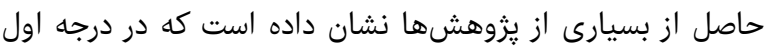

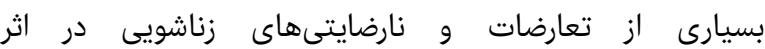

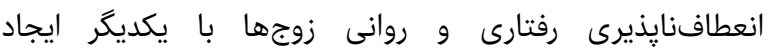

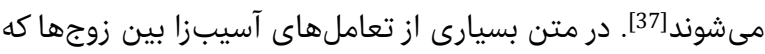

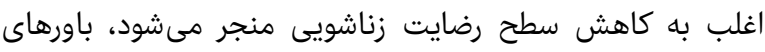

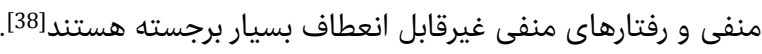

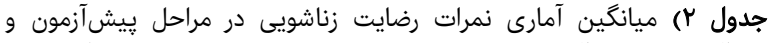

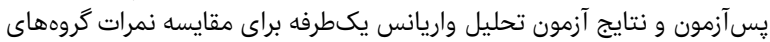

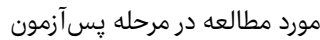

\begin{tabular}{|c|c|c|c|c|c|}
\hline معنىدارى & مقدار F & آزادى & يس آزمون & ييش آزمون & كوردمهالى \\
\hline \multirow{3}{*}{.$/ . * k$} & \multirow{3}{*}{91.41} & \multirow{3}{*}{$r$} & $|\mathrm{~V} / / \Lambda \Delta \pm \mu g /| \Delta$ & $\mid F \& / Y \cdot \pm r V / Q q$ & $\begin{array}{r}\text { Sروه درمان } \\
\text { ACT }\end{array}$ \\
\hline & & & $|V V / T \cdot \pm \mu g /| \varepsilon$ & $\begin{array}{c}I V Q \pm Y N / Y G \\
I Q V\end{array}$ & معنادرمانى \\
\hline & & & $\begin{array}{c}/ F \Delta \pm r q / \mu V \\
\| k r\end{array}$ & $\begin{array}{c}|\varepsilon \Delta \pm \mu| / \mu r \\
\mid \kappa \mu\end{array}$ & كروه كنترل \\
\hline
\end{tabular}

جدول سا) نتايج آزمون تعقيبى توكى براى مقايسه اختلاف ميانكينهاى رضايت

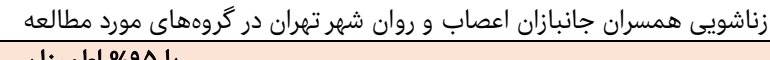

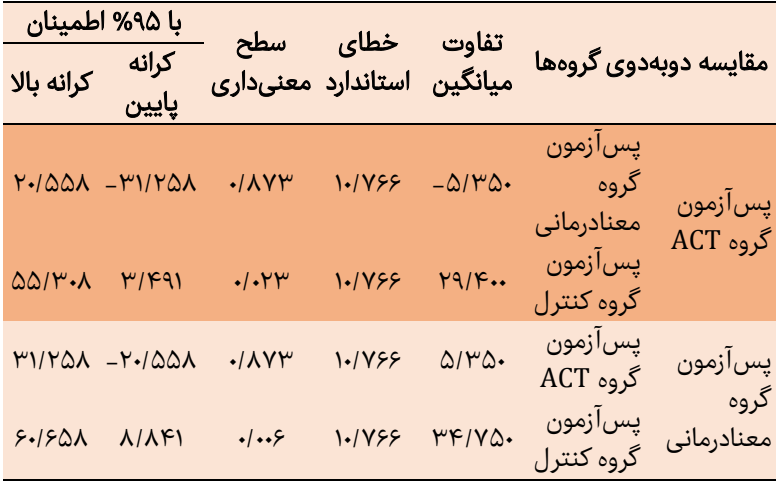

يزوهش حاضر با هدف مقايسه اثربخشى درمان مبتنى بر يذيرش و

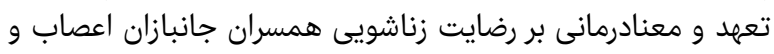

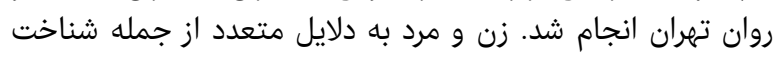

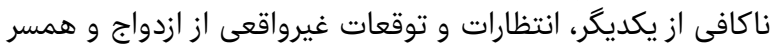

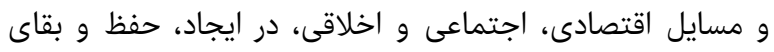

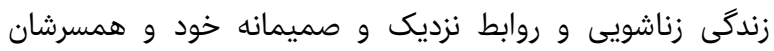

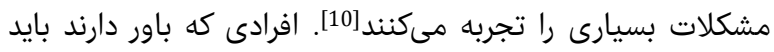

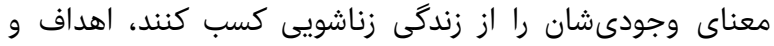

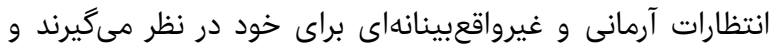

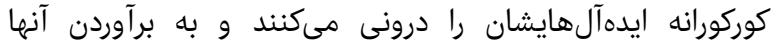

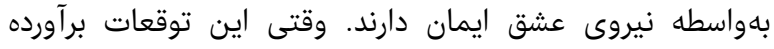

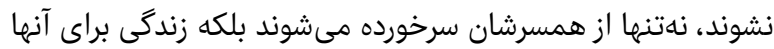

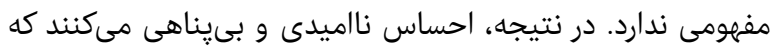

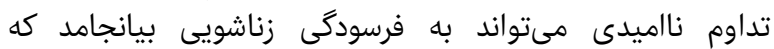

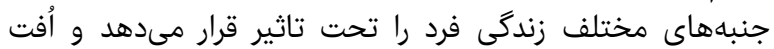

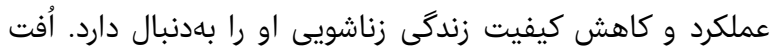

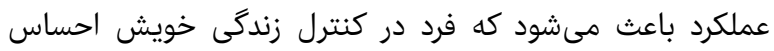

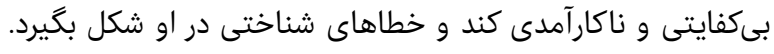

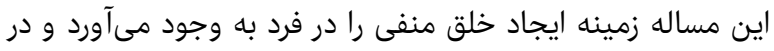

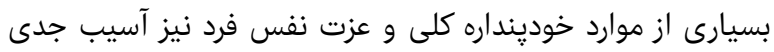

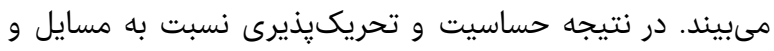

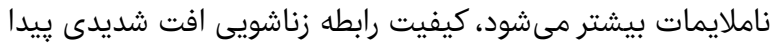

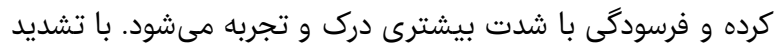

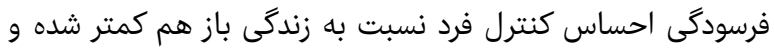

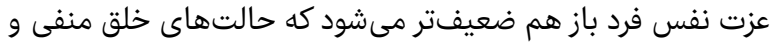

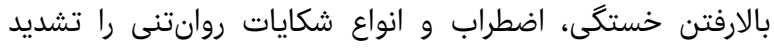

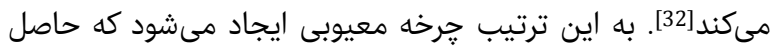

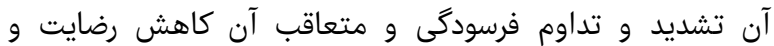


رضايت زناشويى همسران جانبازان اعصاب و روان موثر هستند و

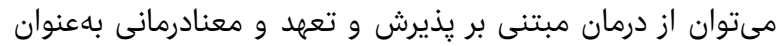
يك اقدام مكمل استفاده نمود.

تشكر و قدردانى: از مسئولان و همكاران محترم مركز دانشيزئوهان إندان

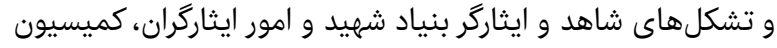

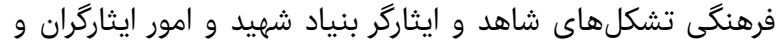

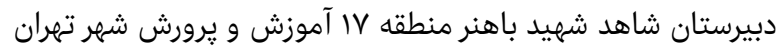

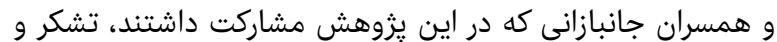

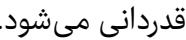
تاييديه اخلاقى: طرح مذكور يس از از ارزيابىهاى اوليه و نكارش

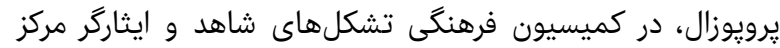

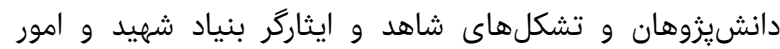

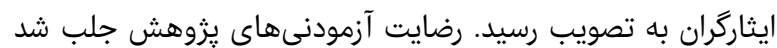

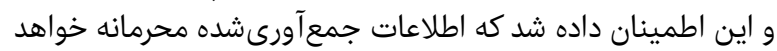

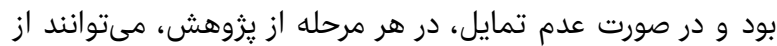

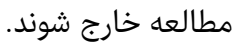

تعارض منافع: تعارض منافع با شخص يا سازمانى وجود ندارد.

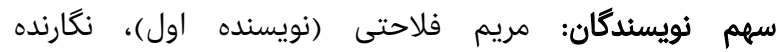

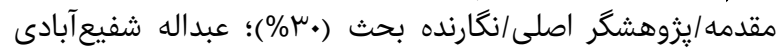

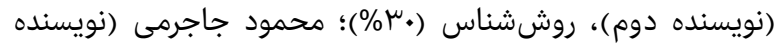

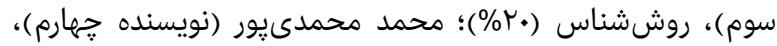

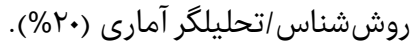

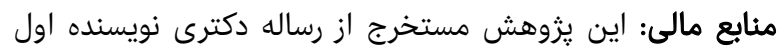

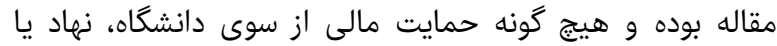

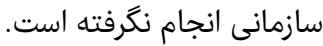

\section{منابع}

1- Askarian S, Hasanzadeh Sablooei MH, Asghari Ebrahimabad MJ. An inquiry into the effect of teaching the skills to confront with difficulties by using an Islamic approach on psychological. Psychol and Relig. 2014;6(4):23-37. [Persian]

2- Askarian S, Asghari MJ, Hassanzadeh MH. Investigating the influence of training religious coping skillson resiliency, adaptability and stress management in veterans' spouses. Iran J War Public Health. 2013;6(1):60-7. [Persian]

3- Ahmadi Kh, Nabipour Ashrafi M, Kimiaee A, Afzali MH. Effect of family problem-soving on marital satisfaction. J Appl Sci. 2010;10(8):682-7.

4- Patrik S, Sells JN, Giordano FG, Tollerud TR. Intimacy, differentiation and personality variables as predictor of marital satisfaction. Fam J. 2007;15(4):359-8.

5- Rosen-grandon JR, Myers JE, Hattie JA. The relationship between marital characteridtics, marital interaction processes and marital satisfaction. J Couns Dev. 2004;82(1):58-68.

6- Bradbury TN, Finchman FD, Beach SR. Research on the nature and determinants of marital satisfaction: A decade in review. J Marriage Fam. 2000;62(4):964-80

7- Juarascio AS, Forman EM, Herbert JD. Acceptance and commitment therapy versus cognitive therapy for the treatment of comorbid eating pathology. Behav Modif. 2010;34(2):175-90.

8- Craske MG, Niles AN, Burklund LJ, Wolitzky-Taylor KB, Vilardaga JC, Arch JJ, et al. Randomized controlled trial of cognitive behavioral therapy and acceptance and

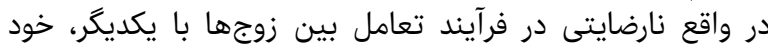

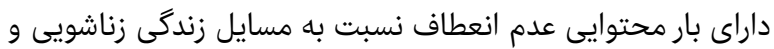

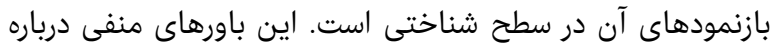

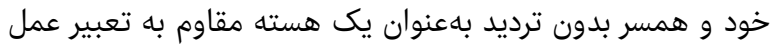

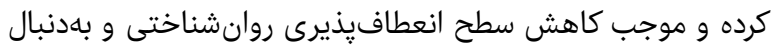

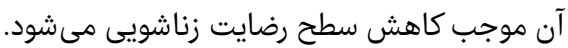

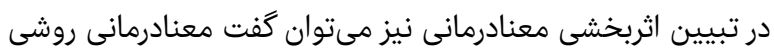

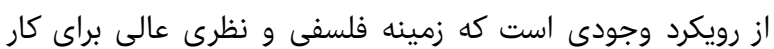

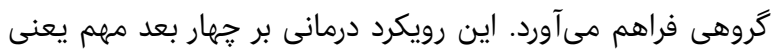

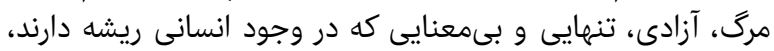

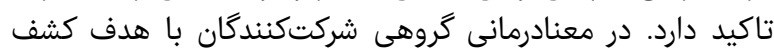

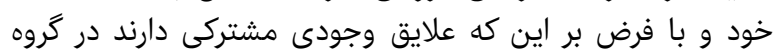

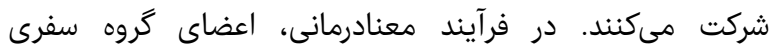

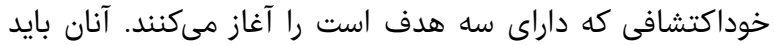

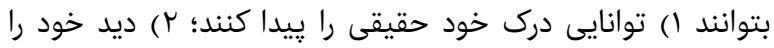

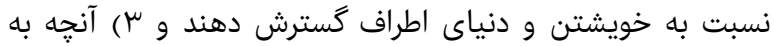

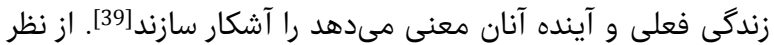

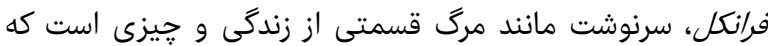

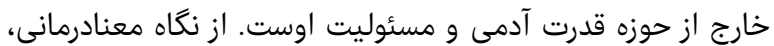

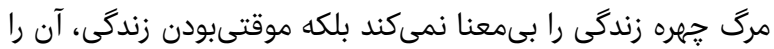

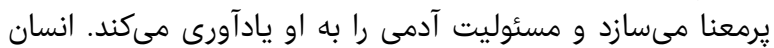

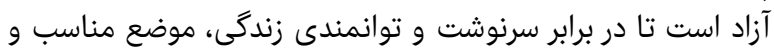

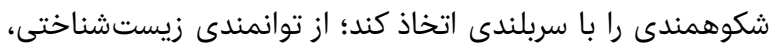

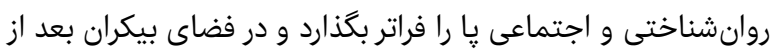

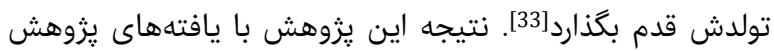

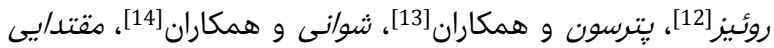

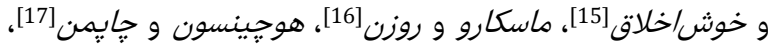

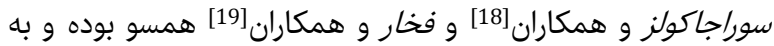

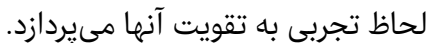

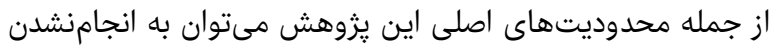

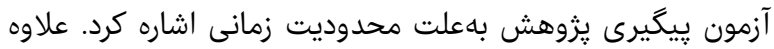

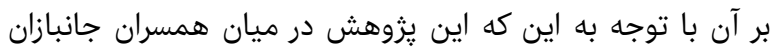

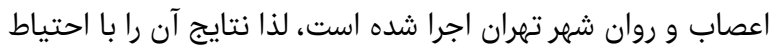

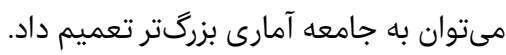

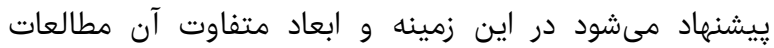

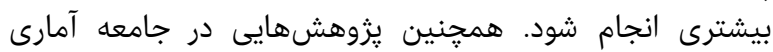

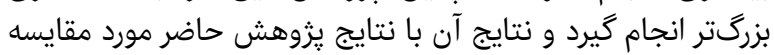

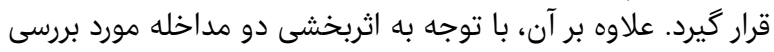

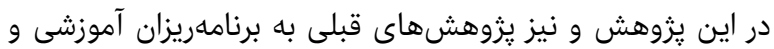

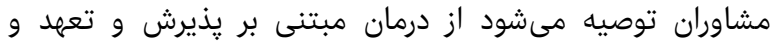

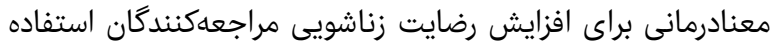

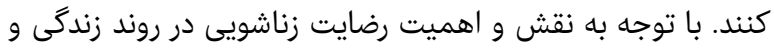

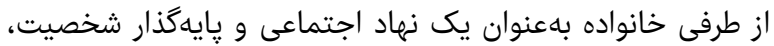

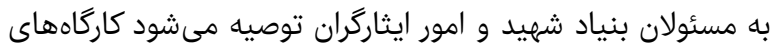

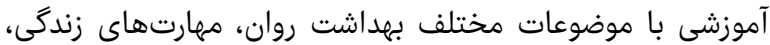

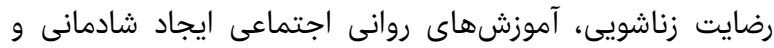

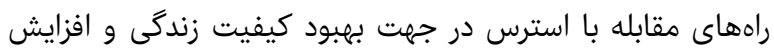

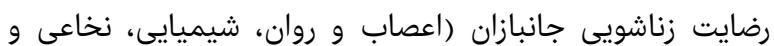
كروههاى خاص)، همسران و فرزندان آنها اجرا نمايند.

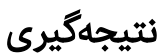

درمان مبتنى بر يذيرش و تعهد و معنادرمانى هر دو بر بهبود 


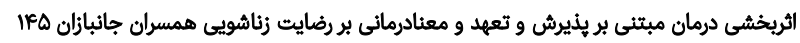
freedman wives. J Appl Couns. 2015;5(2):97-112. [Persian]

24- Cristofolini L, Testoni M. The importance of sample size and statistical power in experimental research: A comparative study. Acta Bioeng Biomech. 2000;2(1):316.

25- Mangeli M, Ramezani T, Mangeli S. The Effect of educating about common changes in pregnancy period and the way to cope with them on marital satisfaction of pregnant Women. Iran J Med Educ. 2009;8(2):305-13. [Persian]

26- Hafezi Kankat H, Ghadami SA. Comparative study of the effect of group counseling with two selection approaches and cognitive-behavioral attitude on couple marital satisfaction in norahabad city. J Couns Res. 2011;10(38):51-80. [Persian]

27- Fowers BJ, Olson DH. Predicting marital success with prepare: Aperdictive validity study. J Marital Fam Ther. 1986;12(4):403-13.

28- Solymanian AA. Effect of irrational thoughts (cognitive approach) on marital satisfaction [Dissertation]. Tehran: Tarbiat Moallem University; 1994. [Persian]

29- Hosseinpour M, Shahrouei S, Ebadi N. Effect of family therapy training on cognitive - behavioral approach on marital satisfaction. J Soc Psychol. 2009;4(11):95-106. [Persian]

30- Hayes SC. Acceptance and commitment therapy, relational frame theory, and the third wave of behavioral and cognitive therapies. Behav Ther. 2004;35(4):639-65. 31- Frankle VE. Man's search for Meaning: An Introduction to logotheraphy. NewYork: Washington square press; 1963.

32- Pirkhaefi A, Salehi F. Effectiveness of group logotherapy on promotion mental health of women with breast cancer. Q J Health Psychol. 2014;2(4):61-9. [Persian]

33- Frankle VE. The doctor and the soul: from psychotherapy to logotherapy. 2nd Edition. New York: Bantam Books; 1965.

34- Hayes SC, Smith S. Get out of your mind and into your life: the new acceptance and commitment therapy (a new harbinger self-help workbook). 1st Edition. Oakland, CA: New Harbinger; 2005.

35- Hayes SC, Strosahl KD. A practical guide to acceptance and commitment therapy. New York: Springer Science and Business Media Inc; 2010.

36- Hayes SC. Climbing our hills: a beginning conversation on the comparison of acceptance and commitment therapy and traditional cognitive behavioral therapy. Clin Psychol. 2008;15(4):286-95.

37- Asarnow JR, Jaycox LH, Tompson MC. Depression in youth: psychosocial interventions. J Clin Child Psychol. 2001;30(1):33-47.

38- Sayers SL, Kohn CS, Fresco DM, Bellack AS, Sarwer DB. Marital cognitions and depression in the context of marital discord. Cogn Ther Res. 2001;25;(6):713-32.

39- Corey G. Theory and practice in group counseling. $4^{\text {th }}$ Edition. Bostson: Brooks/Cole Publishing Company; 1995. commitment therapy for social phobia: outcomes and moderators. J Consult Clin Psychol. 2014;82(6):1034-48. 9- Ost LG. The efficacy of acceptance and commitment therapy: an updated systematic review and metaanalysis. Behav Res Ther. 2014;61(1):105-21.

10- Breitbart W, Gibson CH, Poppito SR, Berg A. Psychotherapeutic interventions at the end of life: a focus on meaning and spirituality. Can J Psychiatry. 2004;49(6):366-72.

11- Steger MF, Frazier P, Oishi S, Kaler M. The meaning in life questionnaire: assessing the presence of and search for meaning in life. J Couns Psychol. 2006;53(1):80-93.

12- Ruiz FJ. A review of acceptance and commitment therapy (ACT) empirical evidence: Correlational, experimental psychopathology, component and outcome studies. Int J Psychol Psychol Ther. 2010;10(1):125-62.

13- Peterson BD, Eifert GH, Feingold T, Davidson S. Using acceptance and commitment therapy to treat distressed couples: a case study with two couples. Cogn Behav Pract. 2009;16(4):430-42.

14- Showani E, Zahrakar K, Rasooli M. Effectiveness of acceptance and commitment therapy on reducing the components of couple burnout in veterans' spouses. Iran J War Public Health. 2016;8(2):111-8. [Persian]

15- Moghtadayi M, Khoshakhlagh H. Effectiveness of acceptance- and commitment-based therapy on psychological flexibility of veterans' spouses. Iran J War Public Health. 2015;7(4):183-8. [Persian]

16- Mascaro N, Rosen DH. The role of exestential meaning as a buffer against stress. J Hum Psychol. 2006;46(2):168-90.

17- Hutchinson GT, Chapman BP. Logotherapy-Enhanced REBT: an integration of discovery and reason. J Contemp Psychother. 2005;35(2):145-55.

18- Sorajjakools S, Aja V, Chilson B, Ramírez-Johnson J, Earll A. Disconnection, depression, and spirituality: A study of the role of spirituality and meaning in the lives of individuals with severe depression. Pastoral Pschol. 2008;56(5):521-32.

19- Fakhar F, Navayinezhad S, Foroughan M. The role of group counseling with logo-therapeutic approach on the mental health of older women. Salmand. 2008;3(1):5867. [Persian]

20- Riebscheger J, Scheid C, Luz C, Mickus M, Liszewski C, Eaton M. How are the experiences and needs of families of individuals with mental illness reflected in medical education guidelines? Acad Psychiatry. 2008;32(2):11926.

21- Hudson P, Quinn K, Kristjanson L, Thomas T, Braithwaite M, Fisher J, Cockayne M. Evaluation of a psycho-educational group program for family caregivers in home-based palliative care. Palliat Med. 2008;22(3):270-80.

22- Mcfarlane WR, Dixson L, Lukens E, Lucksted A. Family psychoeducation and schizophrenia: a review of the literature. J Marital Fam Ther. 2003;29(2):223-45.

23- Mousavi M, Rashidi A, Golmohammadian M. Effectiveness of acceptance and commitment therapy on increasing marital satisfaction of the veteran and 